\title{
Coupling Atomistic and Continuum Models for Multi-scale Simulations of Gas Flows
}

\author{
Vladimir Kolobov, Robert Arslanbekov, and Alex Vasenkov \\ CFD Research Corporation, 215 Wynn Drive, \\ Huntsville, AL , 35803, USA

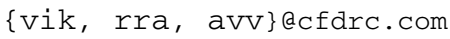

\begin{abstract}
This paper describes two computational tools linking atomistic and continuum models of gaseous systems. The first one, a Unified Flow Solver (UFS), is based on a direct Boltzmann solver and kinetic CFD schemes. The UFS uses an adaptive mesh and algorithm refinement procedure for automatic decomposition of computational domain into kinetic and continuum parts. The UFS has been used for a variety of flow problems in a wide range of Knudsen and Mach numbers. The second tool is a Multi-Scale Computational Environment (MSCE) integrating CFD tools with Kinetic Monte Carlo (KMC) and Molecular Dynamics (MD). The MSCE was applied for analysis of catalytic growth of vertically aligned carbon nanotubes (CNT) in a $\mathrm{C}_{2} \mathrm{H}_{2} / \mathrm{H}_{2}$ inductively coupled plasma. The MSCE is capable of predicting paths for delivering carbon onto catalyst/CNT interface, formation of single wall or multi-wall CNTs depending on the shape of catalyst, and transition from nucleation to the steady growth of CNTs.
\end{abstract}

Keywords: Rarefied Gas Dynamics, Boltzmann solver, Kinetic Monte Carlo, Molecular Dynamics, carbon nanotubes, Unified Flow Solver.

\section{Introduction}

The need for computational analysis at different scales appears in different fields of science and engineering ranging from aerospace applications to material processing. Recent advances of nanotechnology demand understanding of manufacturing processes at atomistic scales for design smart materials and structures. The development of hybrid solvers combining kinetic and fluid models has been an important area of research over the last decade (see Refs. [1,2] for reviews). The challenges of developing multi-scale methods are physical and numerical. Refining computational mesh in continuum simulations down to the atomic scale results in the time step governed by the smallest element of the mesh. Using such small time steps in large cells result in an enormous waste of computing time since continuum dynamics usually evolves on a much larger time scale. Efficient multiscale methods should use different time steps in atomistic and continuum regions. Another major problem in coupling atomistic and continuum models appears in pathological wave reflections at an interface between kinetic and continuum regions, induced by high frequency processes in the kinetic regions. 
This paper describes two computational tools linking atomistic and continuum models for gaseous systems. The first one is a Unified Flow Solver (UFS) for simulations of rarefied, transitional and continuum flows. The UFS solves the Boltzmann and continuum equations in appropriate parts of computational domain. Domain decomposition and coupling algorithm are based on the adaptive mesh and algorithm refinement methods. The computational mesh in physical space is dynamically adapted to the solution and geometry using a tree-based data structure. The UFS separates non-equilibrium and near-equilibrium domains using continuum breakdown criteria and automatically selects appropriate continuum and kinetic solvers. The UFS was used for a variety of flow problems in a wide range of Knudsen and Mach numbers and has recently been extended to multicomponent mixtures of molecular gases with internal degrees of freedom.

The second tool is a Multi-Scale Computational Environment (MSCE) integrating CFD tools with Kinetic Monte Carlo (KMC) and Molecular Dynamics (MD) tools [3]. The atomistic KMC and MD solvers are used in regions where atoms selfassemble into molecular structures. For coupling atomistic and continuum models, macroscopic fluxes from the CFD solver are transferred to KMC, which calculates velocity distributions of gas species near surfaces for nanomaterial fabrication. The MD simulations are used for calculation of surface reaction rates used in KMC. The MSCE was applied for analysis of catalytic growth of vertically aligned carbon nanotubes (CNT) in a $\mathrm{C}_{2} \mathrm{H}_{2} / \mathrm{H}_{2}$ inductively coupled plasma. We have demonstrated that MSCE is capable of predicting paths for delivering carbon onto catalyst/CNT interface, formation of single wall or multi-wall CNTs depending on the shape of catalyst, and transition from nucleation to the steady growth of CNTs [4].

\section{Unified Flow Solver}

The development of hybrid solvers combining kinetic and continuum models for gas flows has been an important area of research over the last decade. The key parameter defining the choice of the appropriate physical model is the local Knudsen number, $K n$, defined as the ratio of the mean free path to the characteristic size of the system. Particle methods such as DSMC or Molecular Dynamics are used in regions with strong deviations from equilibrium, and continuum (Euler or NS) solvers are used elsewhere. Statistical noise inherent to DSMC has been identified as an obstacle for the development of hybrid solvers [5]. Direct methods of solving Boltzmann equation have recently emerged as a viable alternative to the particle methods [6-8]. They are preferable for coupling kinetic and continuum models because similar numerical techniques are used for solving both the Boltzmann and continuum equations. The Unified Flow Solver (UFS) uses the direct Boltzmann solver and kinetic schemes for the continuum solvers to facilitate coupling of the solutions based on continuity of half-fluxes in the finite volume approach.

\subsection{UFS Solution Procedure}

The UFS architecture is described in details in [9]. Here, we only briefly outline some important features. Geometry of the problem is defined using standard files and a 
Cartesian mesh is generated automatically around objects embedded in computational domain. A continuum solver is running first and the computational grid is dynamically adapted to the solution. Kinetic domains are identified using continuum breakdown criteria, and the Boltzmann solver replaces the continuum solver in the kinetic domains. The coupling of kinetic and continuum solvers at the interface occurs seamlessly based on the continuity of half-fluxes.

For solving the Boltzmann equation, a Cartesian mesh in velocity space is introduced with a cell size $\Delta \xi$ and nodes $\xi_{\beta}$. Using this velocity mesh, the Boltzmann equation for each gas component is reduced to linear hyperbolic system of transport equations in physical space with a nonlinear source term

$$
\frac{\partial f_{\beta}}{\partial t}+\nabla_{\mathbf{r}} \cdot\left(\xi_{\beta} f_{\beta}\right)=I\left(f_{\beta}, f_{\beta}\right)
$$

The solution of the system (1) can be found by standard CFD methods for arbitrary mesh in physical space. We use efficient conservative methods of calculating the Boltzmann collision term $I(f, f)$ developed by Tcheremissine [10] using Korobov's sequences [11] for the evaluation of multi-dimensional integrals.

The parallel version of the UFS enforces a dynamic load balance among processors. The procedure of domain decomposition is performed using space-filling curves. Different weights are assigned to kinetic and continuum cells depending on CPU time required for performing computations in the cells [12]. Figure 1 illustrates an example of 3D simulations of the Inflatable Reentry Vehicle Experiment (IRVE) [13] for $K n=0.01$ and $M=3.94$ performed on an 8 node cluster.
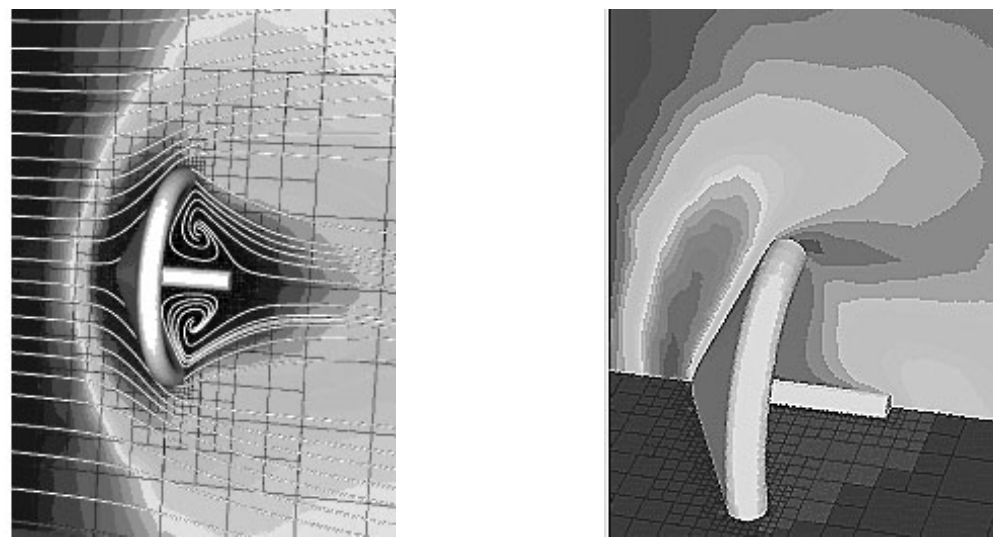

Fig. 1. Streamlines, Mach number, and computational mesh (on the left). Gas temperature in the vertical plane, kinetic (light) and continuum (dark) domains in the horizontal plane (on the right).

\subsection{Molecular Gases and Gas Mixtures}

The UFS has been extended to molecular gases and gas mixtures. Figure 2 shows an example of supersonic flow of a binary mixture over a cylinder at $M=2$, for 3 
Knudsen numbers. Two species with the mass ratio of 2 with no chemical reactions are considered. The Hard Sphere model of molecular interactions is used for the Boltzmann solver. The domain decomposition is performed using continuum breakdown criterion based on the gradient of the total gas density. The temperatures of species become different in the kinetic domains while they are equal in the continuum domains. For these simulations, the typical number of velocity nodes (in each direction) is 15-20 depending on the Mach number and mass ratio of gas species.

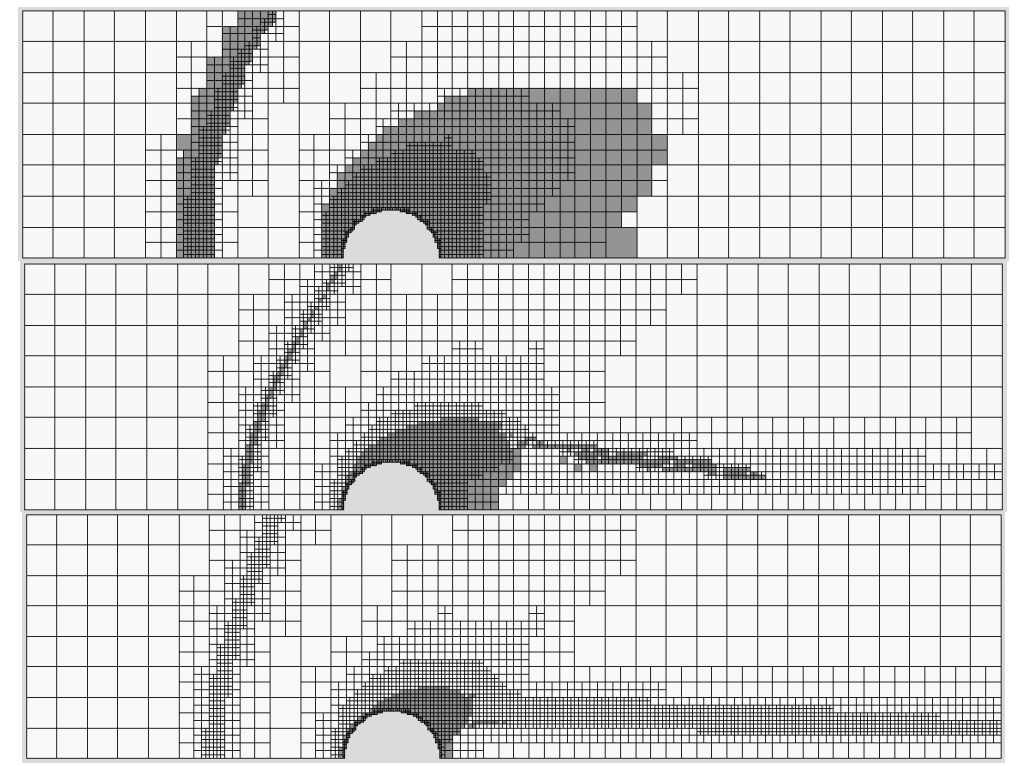

Fig. 2. The computational mesh and kinetic/continuum domains for binary mixture of monatomic gases at $M=2$, for $K n$ numbers $0.125,0.025$, and 0.0125

The kinetic solver has been extended to molecular gases with internal degrees of freedom following the work [14]. Figure 3 shows the shock wave (SW) structure in Nitrogen at $M=12.9$ with the center of SW located at $X=0$. On the left are distributions of gas density, translational and rotational temperatures, on the right are rotational spectra for 25 levels at different points along the wave front. On the $x$-axis is the rotational number; on the $y$-axis is the population of the rotational levels. It is clearly seen that the rotational equilibrium inside the SW does not exist for such a high Mach number. The typical CPU time for these SW simulations is about 30 hours on an AMD64 3000 processor. Although accurate calculations require considerable CPU time that can be reduced by using crude discretization of the velocity space and reduced number of levels of the rotational energy spectrum [15].

We have also extended the kinetic solver for vibrationally excited molecules. For future development of the UFS, we plan to take into consideration that vibrational equilibration in VT and VV collisions occur considerably slower compared to 

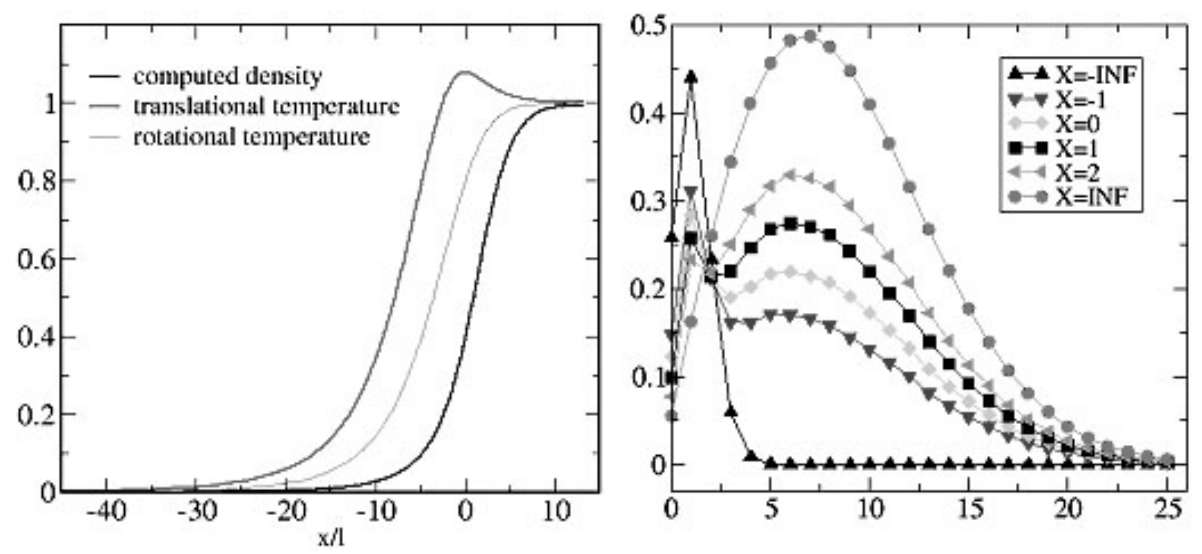

Fig. 3. SW structure in Nitrogen at $M=12.9$ : gas density and temperatures (left) and rotational spectra at different points (right)

translational and rotational equilibration. As a result, in most cases, it is adequate to model computational domains where vibrational relaxation and chemical reactions take place using continuum models.

\section{Multi-scale Models for Computational Material Science}

Computational material science deals with strongly coupled processes occurring at disparate length and time scales at an interface between gaseous and solid phases. The gas-phase processes (chemical reactions, heat transfer, etc.) are typically several orders of magnitude slower than the surface processes. Continuum models or atomistic models are used for simulations of reactive gas flows at the reactor scale. These models are not appropriate for modeling fast surface processes such as surface diffusion of adsorbed molecules, chemical reactions or atomic relaxation occurring with characteristic times of $0.1 \mathrm{~ns}$ and spatial scales of $0.1 \mathrm{~nm}$. Such processes are modeled using atomic-scale models such as Kinetic Monte Carlo (KMC) or Molecular Dynamics (MD). Over the last several years, there has been a rapidly growing interest in the development of computational tools with different levels of detail. The major challenge here is the development of algorithms capable of bridging atomistic and continuum models working on disparate time and length scales.

\subsection{Bridging Disparate Length and Time Scales}

For bridging disparate spatial scales, Kevrekidis et al. [16] introduced the "Gap-tooth" method involving atomistic modeling in tiny teeth, defined as regions where continuum models are not available or are invalid, and a less detailed continuum simulation within large gaps referred to as regions where nanoscale phenomena are not important. This method is designed to approximate a so-called "time-stepper" for an unavailable macroscopic equation in a macroscopic domain. The authors have demonstrated the ability of this model to solve selected multiscale hydrodynamic 
problems such as boundary layer behavior and shock formation. It was also shown that it is possible to obtain a convergent scheme without constraining the microscopic code, by introducing buffer regions around the computational boxes. The Gap-tooth approach was successfully adapted for integrating KMC modeling with CFD reactorscale simulations in the MSCF described in the next section.

Bridging the time scales is an equally difficult and fundamentally different problem than bridging the length scales. The major issue here is that the dynamics of complex systems is often driven by rare but important events [17]. The simulations of these events often require long run times, and rapidly become prohibitively expensive due to the exponential scaling with inverse temperature. Several groups reported significant progress in the development of new techniques for accelerated modeling of rate events [18,19]. Kevrekidis et al. introduced a coarse model for mapping a mesoscopic description onto atomistic description [20]. They assumed that a coarse model (e.g. continuum model) exists in closed form for the fine scale simulator (e.g. KMC model). Based on this assumption, a Coarse Controller was constructed for KMC simulator, and the developed framework was applied for a simple surface catalyst. In subsequent studies, this group further developed the Coarse Controller and used it to accelerate MD modeling. The projection operator formalism was introduced to integrate the dynamics of rare events with fastest molecular processes of bond vibration and atomic collisions [21]. This was achieved by estimating both the thermodynamic driving forces for slow motions and their dynamic properties. Information about the slow coarse dynamics was extracted from the projected motions of many, appropriately initialized, but otherwise independent and unbiased replica simulations. The advantage of the coarse MD is that it can be used for any type of intermolecular potentials and for a wide range of temperatures.

\subsection{Multi-scale Computational Framework}

The Multi-Scale Computational Framework (MSCF) has been developed for material processing applications [3]. The MSCF integrates continuum models with atomistic KMC and MD models, using the coarse timestepper and gap-tooth modules as bridging algorithms. The reactor-scale continuum simulator is used in large gaps where details of atomic motion are not important, while atomistic KMC and MD solvers are applied to tiny regions, teeth, defined as areas where atoms self-assemble into molecular structures. Macroscopic fluxes from the continuum solver are transferred to the gap-tooth module for estimating microscopic fluxes towards the substrate. These microscopic fluxes are used in the KMC solver for prescribing velocity distributions of ions and neutrals at the source plane. The gap-tooth module also accounts for the influence of KMC modeling in one tooth on those in the neighboring teeth by establishing a relationship between incoming microscopic fluxes to each tooth and outgoing fluxes from the neighboring teeth. The velocity distributions of neutral species in KMC are chosen as isotropic Maxwellian distributions; the ions are launched with mono-directional and mono-energetic distributions. The Coarse Timestepper module projects, in time, the rates and probabilities of chemical reactions computed by the MD solver. Figures 4 and 5 illustrate the MSCF application to analysis of catalytic growth of vertically aligned carbon nanotubes (CNT) in a $\mathrm{C}_{2} \mathrm{H}_{2} / \mathrm{H}_{2}$ inductively coupled plasma [4]. 


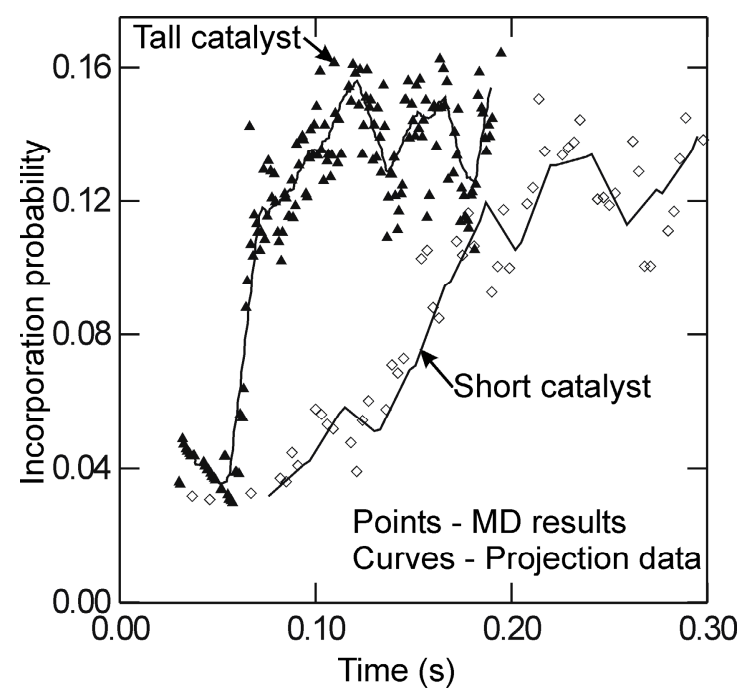

Fig. 4. Transition from nucleation to steady growth of CNTs is illustrated by time-dependent incorporation probabilities for the tall and short catalyst

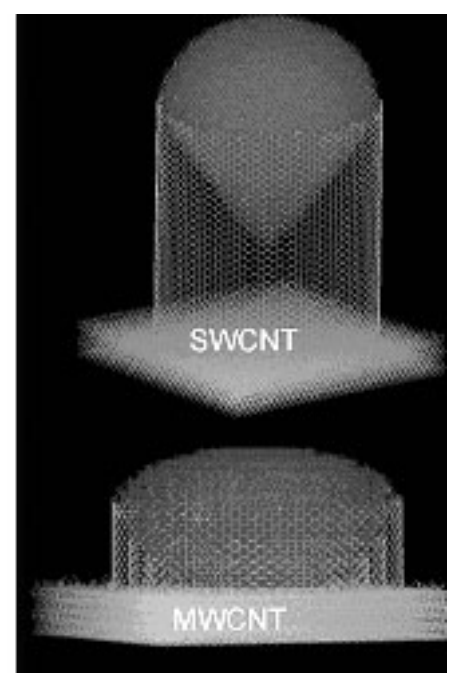

Fig. 5. The growth of single wall and multi-wall CNTs on the catalysts of different shape

\section{Conclusion}

We have described two computational tools linking atomistic and continuum models of gaseous systems. The UFS was originally developed for aerospace applications. We believe that with additional physical models and further improvements, the UFS will be valuable for a wide range of applications from trans-atmospheric flights to low-pressure material processing and semiconductor manufacturing. The MSCF, originally developed for CNT applications, will be further extended into processing of biocompatible polymers and other applications.

Acknowledgments. The UFS was developed in collaboration with Drs. V.V. Aristov, A.A. Frolova, S.A. Zabelok, and F.G. Tcheremissine from the Russian Academy of Sciences. The work is partially supported by USAF and NSF SBIR Projects

\section{References}

1. Wijesinghe, H.S., Hadjiconstantinou, N.G.: A discussion of hybrid atomistic-continuum methods for multiscale hydrodynamics. Int. J. Multiscale Comput. Eng. 2 (2004), 189

2. Kolobov, V.I., Bayyuk, S.A., Arslanbekov R.R., Aristov, V.V., Frolova, A.A., Zabelok, S.A.: Construction of a Unified Continuum/Kinetic Solver for Aerodynamic Problems, AIAA Journal of Spacecraft and Rockets 42 (2005), 598-605

3. Vasenkov, A.V., Fedoseyev, A. I., Kolobov, V. I., Choi, H.S., Hong, K.-H., Kim, K., Kim, J., Lee, H. S., Shin, J. K.: Computational Framework for Modeling of Multi-Scale Processes, J. Comput. Theor. Nanoscience 3 (2006), 453-458 
4. Vasenkov, A.V., Kolobov, V. I.: Modeling of multi-scale processes during the growth of carbon nanotubes, in: Technical Proceedings of the 2006 NSTI Nanotechnology Conference and Trade Show. Vol. 1 (2006), 617-621

5. Carlson, H.A., Roveda, R., Boyd, I.D., Candler, G.V.: A Hybrid CFD-DSMC Method of Modeling Continuum-Rarefied Flows, AIAA Paper 2004-1180 (2004)

6. Rogier, F., Schneider, J.A.: A direct method for solving the Boltzmann equation. Transport Theory Stat. Phys. 23 (1994), 313-338

7. Aristov,V.V.: Direct Methods for Solving the Boltzmann Equation and Study of NonEquilibrium Flows, Kluwer Academic Publishers, Dordrecht, (2001)

8. Tcheremissine, F.G.: Direct Numerical Solution of the Boltzmann Equation, in: M.Capitelli (ed.) Rarefied Gas Dynamics, AIP Conference Proceedings, N.Y. vol. 762 (2005), 677-685

9. Kolobov, V.I., Arslanbekov, R.R., Aristov, V.V., Frolova, A.A., Zabelok, S.A.: Unified solver for rarefied and continuum flows with adaptive mesh and algorithm refinement, J. Comput. Phys. (2006), doi:10.1016/j.jcp.2006.09.021

10. Tcheremissine, F.G.: Solution to the Boltzmann kinetic equation for high-speed flows. Comp. Math. Math. Phys. 46 (2006) 315

11. Korobov, N.M.: Exponential Sums and Their Applications, Springer, (2001), 232p

12. Zabelok, S.A, Aristov, V.V., Frolova, A.A., Kolobov, V.I., Arslanbekov, R.R.: Parallel implementation of the Unified Flow Solver. in: Rarefied Gas Dynamics, AIP Conference Proceedings (2007)

13. Moss, J.N. et al.: Low-Density Aerodynamics of the Inflatable Reentry Vehicle Experiment (IRVE), AIAA Paper 2006-1189 (2006)

14. Tcheremissine, F.G.: Solution of the Wang-Chang-Uhlenbeck master equation. Doklady Physics 47 (2002), 872-875

15. Tcheremissine, F.G., Kolobov, V.I., Arslanbekov, R.R.: Simulation of Shock Wave Structure in Nitrogen with Realistic Rotational Spectrum and Molecular Interaction Potential. in Rarefied Gas Dynamics, AIP Conference Proceedings (2007)

16. Samaey, G., Kevrekidis, I.G., Roose, D.: in Multiscale Modeling and Simulation, Springer, Verlag, (2004), 93

17. Hanggi, P., Talkner, P., and Borkovec, M.: Reaction-rate theory: fifty years after Kramers. Rev. Mod. Phys. 62 (1990), 251-341

18. Sorensen, M. R., Voter, A.F.: Temperature-accelerated dynamics for simulation of infrequent events. J. Chem. Phys. 112 (2000), 9599-9606

19. Dellago, C., Bolhuis, P. G., Chandler, D.: Efficient transition path sampling: Application to Lennard-Jones cluster rearrangements. J. Chem. Phys. 108 (1998), 9236-9245

20. Siettos, C. I., Armaou, A., Makeev, A. G., Kevrekidis, I. G.: Microscopic/stochastic timesteppers and "coarse" control: A KMC example. AIChE Journal 49 (2003), 19221926

21. Sriraman, S., Kevrekidis, I. G., Hummer, G.: Coarse Master Equation from Bayesian Analysis of Replica Molecular Dynamics Simulations. J. Phys. Chem. B 109 (2005), 6479-6484 